\title{
VALIDAÇÃO DE METODOLOGIA ANALÍTICA PARA QUANTIFICAÇÃO DE PIROXICAM EM CÁPSULAS DE GELATINA POR ESPECTROFOTOMETRIA ULTRAVIOLETA (UV)
}

\section{VALIDATION OF ANALYTICAL METHODOLOGY FOR QUANTIFICATION OF PIROXICAM IN GELATIN CAPSULES BY ULTRAVIOLET SPECTROPHOTOMETRY (UV)}

\author{
PEREIRA, Aline; SCHESHOWITSCH, Karin; CRUZ, Ariane; \\ SILVA ${ }^{1}$, Marcos Antonio Segatto; STULZER ${ }^{1}$, Hellen Karine
}

${ }^{1}$ Laboratório de Controle de Qualidade, Departamento de Ciências Farmacêuticas, Centro de Ciências da Saúde, Universidade Federal de Santa Catarina, Campus Trindade, 88040-900, Florianópolis, SC, Brasil.

RECEBIDO: JUN/07 ACEITE: AGO/07

\begin{abstract}
RESUMO
A validação é essencial para definir se uma metodologia desenvolvida está completamente adequada aos objetivos a que se destina, a fim de se obter resultados confiáveis que possam ser satisfatoriamente interpretados. Além disso, pode ser considerada um dos principais instrumentos de garantia da qualidade. Foram avaliados os parâmetros de especificidade, seletividade, limite de quantificação, linearidade, intervalo, precisão (repetibilidade e precisão intermediária) e exatidão. A linearidade apresentou coeficiente de correlação igual a 0,99975 no intervalo de $2-8 \mu \mathrm{g} / \mathrm{mL}$. Os excipientes contidos na formulação não são absorvidos no comprimento de onda utilizado, não interferindo assim na análise e quantificação da substância ativa. A quantificação de piroxicam em cápsulas de gelatina através de espectrofotometria UV no comprimento de $333 \mathrm{~nm}$ foi aprovada em todos os parâmetros analisados, sendo portanto, devidamente validada.
\end{abstract}

Palavras-chave: validação de metodologia analítica; espectrofotometria ultravioleta; piroxicam.

\begin{abstract}
The validation is essential to define if a developed methodology is completely adjusted to the objectives that the one is destines, in order to get trustworthy results that can be satisfactorily interpreted. Moreover, it is considered one of the main instruments of quality control. The parameters used in the validation process were: specificity, selectivity, quantification limit, linearity, range, precision (repeatability and intermediate precision) and accuracy. The linearity in the range of 2-8 $\mu \mathrm{g} / \mathrm{mL}$ presented a correlation coefficient of 0.99975 . The excipients in the formulation did not interfere with the analysis and the recovery was quantitative. The quantification of piroxicam in gelatin capsules through UV spectrophotometry in the length of $333 \mathrm{~nm}$ was approved in all the analyzed parameters, being therefore, duly validated.
\end{abstract}

Key words: validation of analytical methodology; ultraviolet spectrophotometry; piroxicam. 


\section{INTRODUÇÃO}

O processo de validação é essencial para definir se uma metodologia desenvolvida está completamente adequada aos objetivos a que se destina, a fim de se obter resultados confiáveis que possam ser satisfatoriamente interpretados. Envolve o desenvolvimento de um método analítico, de uma adaptação ou implementação de um método conhecido e um processo de avaliação que estime sua eficiência [1]. Além disso, pode ser considerada um dos principais instrumentos de garantia da qualidade, pois possibilita o conhecimento das limitações e da confiabilidade de uma metodologia analítica, da instalação de um equipamento ou de um processo produtivo [1,2].

A quantificação de um fármaco em uma formulação é considerada um aspecto vital da garantia da qualidade de medicamentos [2]. Segundo a Farmacopéia Americana [3], a determinação do teor de piroxicam (4-hidroxi2-metil-N-2-piridinil-2H-1,2-benzotiazina-3-carboxa-mida-1,1-dioxida) (figura 1) em cápsulas pode ser realizada através da técnica de cromatografia líquida. Porém, trata-se de uma técnica onerosa que exige grande quantidade de reagentes e materiais, além de um maior tempo de execução. Por esse motivo, o presente trabalho propõe uma técnica alternativa menos dispendiosa e de rápida execução, a espectrofotometria ultravioleta. Entretanto, quando se desenvolve uma nova metodologia analítica é necessário realizar a validação da mesma, para que assim possa ser utilizada com segurança.

O piroxicam é um fármaco que pertence à classe dos antiinflamatórios não esteroidais e é amplamente utilizado no tratamento de artrite reumatóide, osteoartrite, artrite gotosa aguda, condrocalcinose, espondilite anquilosante e inflamação não-reumática $[4,5,6,7,8,9,10,11]$.<smiles>CN1C(C(=O)Nc2ccccn2)=C(O)c2ccccc2S1(=O)=O</smiles>

FIGURA 1: Fórmula estrutural do Piroxicam [12].

Os parâmetros escolhidos para validação realizada no presente trabalho estão de acordo com a Resolução $N^{\circ}$ 899, de 29 de Maio de 2003 [13] e com as recomendações da ICH (The International Conference on Harmonisation of Technical Requirements for Registration of Pharmaceuticals for Human Use) [14], sendo eles: especificidade, seletividade, limite de quantificação, linearidade, intervalo, precisão (repetibilidade e precisão intermediária) e exatidão.

\section{MATERIAIS E MÉTODOS}

\subsection{MATERIAIS}

A substância química de referência (SQR) utilizada como padrão secundário, foi piroxicam com teor declarado de 99,9\% (lote PRX2004026, China).

As cápsulas de piroxicam $20 \mathrm{mg}$ foram adquiridas em três diferentes farmácias de manipulação localizadas no município de Florianópolis, SC, Brasil e foram denominadas formulações A, B e C, sendo suas composições estão descritas na tabela 1. Os reagentes utilizados, álcool etílico, ácido clorídrico e cloreto de sódio apresentavam grau analítico. 
TABELA 1: Composição das formulações A, B e C.

\begin{tabular}{cccc}
\hline Excipientes & Formulação A & Formulação B & Formulação C \\
\hline Piroxicam & $20 \mathrm{mg}$ & $20 \mathrm{mg}$ & $20 \mathrm{mg}$ \\
Estearato de magnésio & $1 \%$ & --- & $0,5 \%$ \\
Aerosil & $1 \%$ & --- & $1 \%$ \\
Lauril sulfato de sódio & --- & $1 \%$ & $1,5 \%$ \\
Celulose microcristalina & $10 \%$ & --- & --- \\
Talco farmacêutico & --- & --- & $30 \%$ \\
Amido de milho & $88 \%$ & $99 \%$ & $67 \%$ \\
\hline
\end{tabular}

\subsection{MÉTODOS}

\subsubsection{ESPECIFICIDADE E SELETIVIDADE}

Nos estudos de especificidade e seletividade do método para determinação do teor de piroxicam, foram realizadas duas varreduras entre 200 e $400 \mathrm{~nm}$ em espectrofotômetro UV modelo Cary 50 BIO - Varian, sendo uma varredura com uma solução padrão de piroxicam $8 \mu \mathrm{g} / \mathrm{mL}$ e outra com uma solução contendo somente os excipientes utilizados nas cápsulas. As soluções foram preparadas dissolvendo-se o conteúdo pesado em $20 \mathrm{~mL}$ de álcool etílico e quantidade de ácido clorídrico $0,5 \mathrm{M}$ suficiente para completar o volume do balão utilizado.

\subsubsection{LINEARIDADE E INTERVALO}

A linearidade do método foi verificada através da leitura em espectrofotômetro na região do UV (333 nm) de soluções de padrão secundário de piroxicam num intervalo que compreende concentrações entre 2 - $8 \mu \mathrm{g} /$ $\mathrm{mL}$. Através dos valores de absorbância obtidos, construiu-se um gráfico de concentração versus absorbância utilizando-se como critério de aceitação um coeficiente de correlação $\left(R^{2}\right)$ maior ou igual a 0,99. A partir dessa curva de calibração, calculou-se o coeficiente angular da reta e o seu ponto de intersecção no eixo y.

\subsubsection{LIMITES DE QUANTIFICAÇÃO}

O limite de quantificação (LQ) (equação 1) é baseado no desvio padrão $(\sigma)$ da resposta e no coeficiente angular (S) da curva de calibração conforme a equação, $L Q=\left(\frac{10 \sigma}{S}\right)$.

\subsubsection{PRECISÃo}

Para o preparo da amostra $50 \mathrm{mg}$ do padrão secundário de piroxicam foram pesados e dissolvidos em $20 \mathrm{~mL}$ de álcool etílico e quantidade suficiente de ácido clorídrico $0,5 \mathrm{M}$ para completar o volume de um balão volumétrico de $100 \mathrm{~mL}$. A partir dessa solução foram preparadas, através de diluições, seis replicatas contendo piroxicam na concentração de $6 \mu \mathrm{g} / \mathrm{mL}$. Este parâmetro foi avaliado em dois níveis:

- Repetibilidade (precisão intracorrida)

Arepetibilidade do método foi determinadaatravés de 3 repetições, contendo seis replicatasa $100 \%$ da concentração do teste (conforme descrito no item preparo da amostra), realizadas pelo mesmo analista, no mesmo laboratório, em períodos alternados (manhã, tarde e noite), verificando se os resultados encontram-se dentro da máxima diferença aceitável. 
- Precisão intermediária

A precisão intermediária foi expressa através de variações dentro do mesmo laboratório, em dias diferentes e por analistas diferentes. $O$ ensaio foi realizado com 6 replicatas a $100 \%$ da concentração do teste (conforme descrito no item preparo da amostra), em 2 dias diferentes com 2 analistas diferentes.

\subsubsection{EXATIDÃO}

Para averiguar a exatidão do método utilizou-se o método de adição de padrão. Quantidades conhecidas de diferentes concentrações do padrão secundário de piroxicam foram adicionadas a concentrações preparadas com as cápsulas manipuladas do fármaco em questão, como mostra a tabela 2.

TABELA 2: CONCENTRAÇÕES DE PADRÃO SECUNDÁRIO DE PIROXICAM ADICIONADOS Á CONCENTRAÇÕES PREPARADAS COM CÁPSULAS MANIPULADAS DE PIROXICAM.

\begin{tabular}{cccc}
\hline Balão & {$[\mathbf{c p s}](\mu \mathrm{g} / \mathrm{mL})$} & [ padrão $\left.2^{\circ}\right](\mu \mathrm{g} / \mathrm{mL})$ & [ total] $(\mu \mathrm{g} / \mathrm{mL})$ \\
\hline 1. & 2 & 1 & 3 \\
2. & 2 & 2 & 4 \\
3. & 2 & 5 & 7 \\
4. & 2 & --- & 2 \\
5. & --- & 8 & 8 \\
\hline
\end{tabular}

Nota: [ ] - concentração; cps - cápsulas.

Após a preparação das soluções mediu-se a absorbância das mesmas no espectrofotômetro na região do UV em $333 \mathrm{~nm}$. A exatidão foi expressa pela relação entre a concentração média determinada experimentalmente e a concentração teórica correspondente, multiplicados por 100. Todo o procedimento foi realizado em triplicata.

\subsection{ANÁLISE ESTATÍSTICA}

A análise estatística dos dados foi realizada através de análise de variância ANOVA unifatorial, no qual os resultados são considerados significativos quando a probabilidade é inferior a $5 \%$ ( $p<0,05 /$ intervalo de confiança de 95 \%) e teste t de Student com nível de significância $\alpha=0,05$ (intervalo de confiança de 95 \%).

\section{RESULTADOS E DISCUSSÃO}

\subsection{ESPECIFICIDADE E SELETIVIDADE}

Especificidade e seletividade é a capacidade que o método possui de medir exatamente um composto em presença de outros componentes, tais como, impurezas, produtos de degradação e componentes da matriz $[3,13,14]$. Porém, no presente trabalho foi avaliado somente a influência dos componentes da matriz. Os resultados para especificidade demonstraram que o método é adequado em relação ao parâmetro avaliado, pois não ocorreu pico de absorção dos excipientes na faixa de luz próximo a $333 \mathrm{~nm}$ (figura 2), comprimento de onda de absorção máxima do piroxicam. 


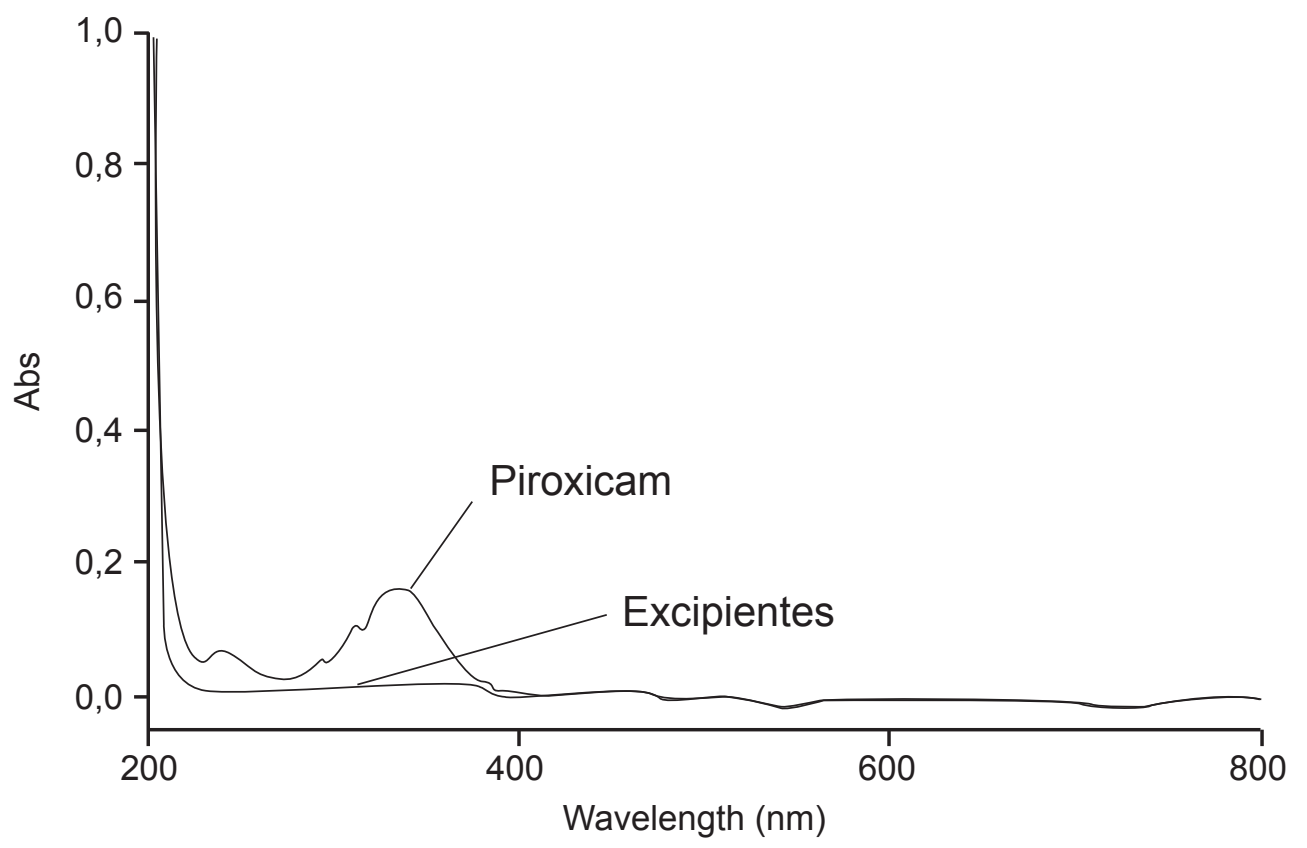

FIGURA 2: Espectro de absorção na região do UV/VIS da mistura de excipientes e de padrão secundário de piroxicam.

\subsection{LIMITE DE QUANTIFICAÇÃO}

Através da análise de regressão linear determinou-se o desvio padrão da linha de regressão, através da equação descrita no item 2.2.3. O limite de quantificação foi estabelecido por meio de análises das soluções contendo concentrações decrescentes de piroxicam até o menor nível determinável com precisão e exatidão aceitáveis, o valor calculado para o limite de quantificação foi de $1,2 \mu \mathrm{g} / \mathrm{mL}$.

\subsection{LINEARIDADE E INTERVALO}

A linearidade de uma metodologia analítica é a capacidade de demonstrar que os resultados obtidos são proporcionais à concentração do analito dentro de um intervalo especificado. Sendo este, a faixa entre os limites de quantificação superior e inferior de um método analítico. [1,13].

A curva de calibração deve apresentar os dados estatísticos de intersecção, da equação da regressão linear, o coeficiente de correlação e a concentração conhecida do analito. Esta pode ser construída usando-se, no mínimo, cinco valores de concentração enquadrados no intervalo definido e é usada para calcular a concentração do fármaco nas amostras, utilizando-se a mesma matriz biológica proposta para o estudo. A suposição clássica da curva de calibração é que a resposta instrumental está linearmente relacionada com a concentração do padrão. O critério mínimo de aceitação do coeficiente de correlação $\left(R^{2}\right)$ foi alcançado igual a 0,99975 [1,13]. As soluções preparadas, dentro do intervalo especificado, apresentaram leituras de absorção diretamente proporcionais à concentração do analito, confirmando a linearidade do método. 
FIGURA 3: CURVA DE CALIBRAÇÃO DEMONSTRANDO A LINEARIDADE E FAIXA DO MÉTODO DE QUANTIFICAÇÃO DO PIROXICAM POR UV.

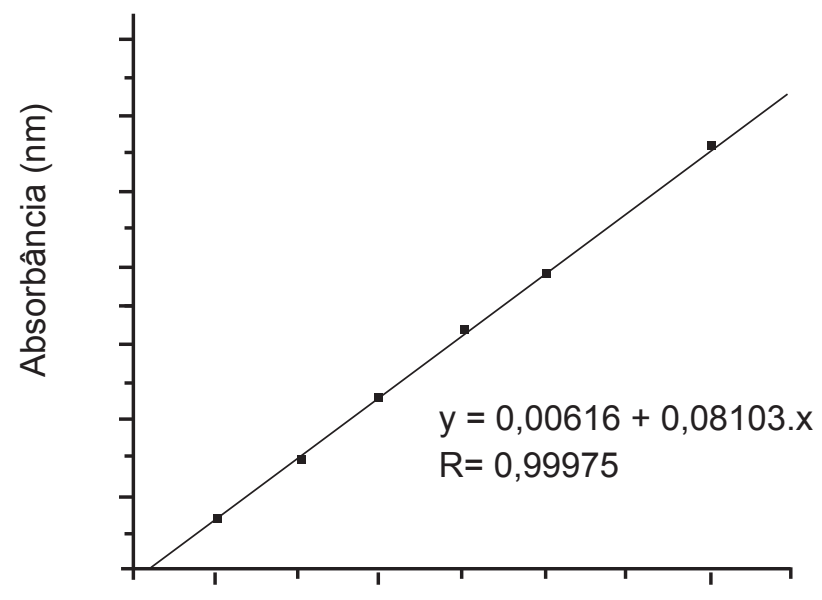

Concentração $(\mu \mathrm{g} / \mathrm{mL})$

De acordo com a figura 3, observa-se que o método apresentou linearidade no intervalo da concentração testada. Determinou-se que o coeficiente angular (A) corresponde a 0,08103 e o valor do intercepto obtido (B) foi diferente de zero.

\subsection{PRECISÃO}

A precisão é a avaliação da proximidade dos resultados obtidos em uma série de medidas de uma mesma amostra. Pode ser classificada em três níveis: repetibilidade, precisão intermediária e reprodutibilidade. Para a validação da metodologia em questão, somente os parâmetros de repetibilidade (concordância entre os resultados dentro de um curto período de tempo com o mesmo analista e mesma instrumentação) (tabela 3) e precisão intermediária (concordância entre os resultados de um mesmo laboratório, mas obtidos em dias diferentes, com analistas diferentes e/ou equipamentos diferentes) (tabela 4) foram avaliados [13].

TABELA 3: ENSAIO DE REPETIBILIDADE (PRECISÃO INTRACORRIDA).

\begin{tabular}{cccc}
\hline & Balão & Absorbância $(\mathrm{nm})$ & Resultado (\%) \\
\hline & 1 & 0,5169 & 95,94 \\
Repetibilidade & 2 & 0,5161 & 96,12 \\
(manhã) & 3 & 0,4991 & 92,62 \\
& 4 & 0,5019 & 93,15 \\
& 5 & 0,5007 & 92,92 \\
& 6 & 0,4989 & 92,60 \\
& 1 & 0,4575 & 92,27 \\
& 2 & 0,4667 & 94,12 \\
\hline
\end{tabular}




\begin{tabular}{cccc}
\hline & Balão & Absorbância (nm) & Resultado (\%) \\
\hline Repetibilidade & 3 & 0,4628 & 93,33 \\
(meio-dia) & 4 & 0,4744 & 95,68 \\
& 5 & 0,4608 & 92,93 \\
& 6 & 0,4731 & 95,42 \\
\hline Repetibilidade & 1 & 0,4671 & 94,20 \\
(tarde) & 2 & 0,4684 & 94,47 \\
& 3 & 0,4724 & 95,28 \\
& 4 & 0,4679 & 94,37 \\
& 5 & 0,4682 & 94,43 \\
& 6 & 0,4734 & 95,48 \\
\hline
\end{tabular}

O resultado do ensaio de repetibilidade foi expresso como desvio padrão relativo (DPR). O DPR calculado foi de $0,48 \%$, o que demonstra que o método não sofre influências do período em que é realizado, uma vez que o DPR máximo permitido pela legislação é de $5 \%$ (RDC nº 899 de 29 de maio de 2003). Aplicou-se também, a análise de variância (ANOVA) para verificar a existência de diferenças significativas entre os períodos de realização do ensaio. $O$ valor de $F$ calculado $(F=0,74 ; p=0,49)$ ficou abaixo do $F$ crítico $(3,68)$, confirmando a eficiência do método quanto à precisão intracorrida.

TABELA 4: ENSAIO DE REPETIBILIDADE (PRECISÃO INTERMEDIÁRIA).

\begin{tabular}{cccc}
\hline & Balão & Absorbância $(\mathrm{nm})$ & Resultado (\%) \\
\hline \multirow{3}{*}{ Analista 1 } & 1 & 0,5169 & 95,94 \\
& 2 & 0,5161 & 96,12 \\
& 3 & 0,4991 & 92,62 \\
& 4 & 0,5019 & 93,15 \\
& 5 & 0,5007 & 92,92 \\
Analista 2 & 6 & 0,4989 & 92,60 \\
& 1 & 0,4711 & 95,02 \\
& 2 & 0,4805 & 96,85 \\
& 3 & 0,4696 & 94,72 \\
& 4 & 0,4638 & 93,53 \\
& 5 & 0,5000 & 100,87 \\
& 6 & 0,4747 & 95,75 \\
\hline
\end{tabular}

O resultado do ensaio de precisão intermediário também foi expresso como desvio padrão relativo (DPR). O DPR calculado foi de 1,66 \%, sendo o DPR máximo permitido igual a $5 \%$ (RDC nº 899 de 29 de maio de 2003). Aplicou-se, também, a análise de variância (ANOVA) para verificar a existência de diferenças estatisticamente significativas entre os ensaios de precisão intermediário. $O$ valor de $F$ calculado $(F=3,18 ; p=0,11)$ ficou abaixo do $F$ crítico $(4,96)$. Através da observação dos resultados obtidos é possível concluir que o método apresenta precisão intercorrida, garantindo a precisão dos resultados independente de quem execute o método. 


\subsection{EXATIDÃO}

Através do parâmetro de exatidão é possível comprovar a proximidade dos resultados obtidos pelo método em estudo, em relação ao valor verdadeiro. Nos casos em que amostras de todos os componentes do medicamento estão disponíveis, se aceita a análise pelo método de adição do padrão, no qual se adicionam quantidades conhecidas do padrão de referência aos excipientes da formulação [13]. A tabela 5 apresenta os valores encontrados para recuperação do piroxicam nas diferentes concentrações.

TABELA 5: RECUPERAÇÃO DO PIROXICAM ADICIONADA À FORMULAÇÃO (EXCIPIENTES) E ANALISADA PELO MÉTODO PROPOSTO.

\begin{tabular}{cccccc}
\hline Balão & $\begin{array}{c}\text { Volume de } \\
\text { piroxicam - } \\
\text { cápsula } \\
\mathbf{( 2 0} \boldsymbol{\mu g} / \mathbf{m L})\end{array}$ & $\begin{array}{c}\text { Volume do } \\
\text { padrão } \mathbf{2}^{\mathbf{0}} \text { de } \\
\text { piroxicam } \\
\mathbf{\mu g} / \mathbf{m L})\end{array}$ & $\begin{array}{c}\text { Concentração de } \\
\text { piroxicam final }(\boldsymbol{\mu} \\
\mathbf{g} / \mathbf{m L})\end{array}$ & $\begin{array}{c}\text { Absorbância } \\
\text { média }(\mathbf{n m})\end{array}$ & $\begin{array}{c}\text { Recuperação } \\
\mathbf{( \% )}\end{array}$ \\
\hline $1-50 \mathrm{~mL}$ & 2,5 & 2,0 & 3 & 0,2476 & 99,61 \\
$2-50 \mathrm{~mL}$ & 5,0 & 2,0 & 4 & 0,3250 & 98,19 \\
$3-10 \mathrm{~mL}$ & 1,0 & 1,0 & 7 & 0,5850 & 101,18 \\
$4-10 \mathrm{~mL}$ & 1,0 & --- & 2 & 0,1598 & 96,17 \\
$5-25 \mathrm{~mL}$ & --- & 4,0 & 8 & 0,6811 & 103,11 \\
\hline
\end{tabular}

A porcentagem média para o ensaio de recuperação foi de $99,65 \pm 1,19 \%$, resultado que traduz a concordância com o valor verdadeiro da amostra. A análise de variância (ANOVA) permite verificar, ainda, a existência de diferença significativa, entre as porcentagens de recuperação, no ensaio que avalia a exatidão da metodologia. $O$ valor de $F$ calculado $(F=0,08 ; p=0,78)$ ficou abaixo do $F$ crítico $(5,32)$, o que reflete a eficiência do ensaio, pois não houve diferença significativa entre as porcentagens de piroxicam recuperadas.

\section{CONCLUSÕES}

A validação da metodologia analítica em estudo demonstrou-se apropriada para a finalidade pretendida, pois pode ser considerada específica, seletiva, linear, precisa e exata. Dentro do intervalo estudado, a metodologia demonstrou ser reprodutível e adequada ao controle de qualidade de formulações que contenham piroxicam. Além disso, trata-se de uma metodologia simples, envolvendo poucas etapas de preparação de amostras e reagentes, pouco dispendiosa e rápida, comparada com a metodologia de determinação de teor descrita na Farmacopéia Americana. 


\section{REFERÊNCIAS}

N. M. BRITO, O. P. A. JUNIOR, L. POLESE, M. L. RIBEIRO, Ecotoxicol. e Meio Ambiente. 13 (2003) 129 - 146.

C. B. BARROS, Revista Biológico. 64 (2002) 175 - 177.

THE UNITED STATES PHARMACOPEIA 26. ed., Rockville: United States Pharmacopeial Convention, 2003. Cd-room.

R. F. BORNE, Nonsteroidal Anti-inflammatory Agents, In: D. A. WILLIAMS, T. L. LEMKE, Foye's principles o medicinal chemistry, EUA, $5^{\text {th }}$ edn., 2002, $751-793$.

A. BURKE, E. SMYTH, G. A. FITZGERALD, Analgesic-Antipyretic agents; Pharmacotherapy of Gout. In: L. L. BRUNTON, J. S. LAZO, K. L PARKER, Goodman \& Gilman's The Pharmacological Basis of Therapeutics, New York, 11th edn., 2006,671 -715 .

A. KOROLKOVAS, Dicionário Terapêutico Guanabara, Rio de Janeiro, 1994/1995 edn., 1994, 21 - 21.6.

C. F. L. LACY, L. L. ARMSTRONG, M. P. GOLDMAN, L. L. LANCE, Drug Information Handbook, Hudson - Cleveland, $8^{\text {th }}$ edn., 2000/01.

MARTINDALE: Sweetman S (Ed), Martindale: The Complete Drug Reference, London, 33 ${ }^{\text {rd }}$ edn., Electronic version In: Micromedex® Healthcare Series, Greenwood Village, Colorado , 2007.

A. C. MOFFAT, M. D. OSSELTON, B. WIDDOP, Clarke's Analysis of Drugs and Poisons: in pharmaceutics, body fluids and postmortem material, London, $3^{\text {rd }}$ edn., 2004, $1463-1464$.

[10] G. PARISOTTO, S.J. de SOUZA, F.M. FERRÃO, C.J. FURTADO, F.R. MOLZ, Revista Brasileira de Ciências Farmacêuticas, 41(4) (2005) $499-505$.

R.B. RAFFA, Analgesic, antipyretic and anti-inflamatory drugs, In: P. BERINGER, A. DERMARDEROSIAN, L. FELTON, S. GELONE, A. R. GENNARO, P. K. GUPTA, J. E. HOOVER, N. G. POPOVICK, W.J. Jr. REILLY, R.H. CHAIR, Remington: the science and practice of pharmacy, Philadelphia $21^{\text {st }}$ edn., 2006, $1524-1542$.

F. VRECER, M. VRBINC, A. MEDEN, International Journal of Pharmaceutics, 256 (2003) 3- 15.

BRASIL. Ministério da Saúde. Agência Nacional de Vigilância (ANVISA) RE n 899, de 29 de maio de 2003, Guia para validação de métodos analíticos e bioanalíticos. Lex: Disponível em: < http://www.anvisa.gov.br>.

$\mathrm{ICH}$ - "International Conference on Harmonization of Technical Requeriments for registration of Pharmaceutical for Human use: Q 2B- validation of Analytical procedure: methodology", 1996. 